\title{
Study of the impact of the food biopolymer system on the clinical-biochemical status of white inbred mice in the invivo experiment
}

\author{
Tatyana Alekseeva ${ }^{*}$, Mihail Korystin ${ }^{1}$, Ekaterina Klimova $^{1}$, Yuliya Kalgina $^{1}$, Lidiya Vitruk $^{2}$, and Lyudmila Malakova ${ }^{1}$ \\ ${ }^{1}$ Department of Service and Restaurant Business, Voronezh State University of Engineering, Voronezh, Russia \\ ${ }^{2}$ Department of Foreign Languages, Voronezh State University of Engineering, Voronezh, Russia
}

\begin{abstract}
Studies have been conducted on the effect of the food biopolymer composition on the clinicalbiochemical status of white inbred mice. The experiment showed that the development of laboratory animals was carried out without abnormalities with the complete safety of all individuals. Clinicalbiochemical dynamics, glucose monitoring and the results of experiments in the exchange of macro and microelements of plasma of mouse blood indicated the normal flow of metabolic processes. In addition, the animals that consumed food additives had a higher rate of absorption of feed carbohydrates and a predominance of osteosynthesis processes in animal bone tissue. The studies conducted allow us to conclude that enrichment and trace elements increase the availability of trace elements, as well as activate the formation and strengthening of the bone tissue of the body.
\end{abstract}

\section{Introduction}

One of the main tasks of the food industry is the creation of commercial food lines to correct the nutritional status of the human body. We have developed the component composition and the technology of biopolymer composition on the basis of domestic raw materials of deep processing for food enrichment. The enrichment composition included albumin of animal origin, bagasse wheat germ and white bean seeds. The purpose of the work was to conduct studies on the influence of the developed food system on the clinical and biochemical status of laboratory animals. Similarity in the structure and functioning of the digestive system (stomach, small and large intestines, liver) and the white mouse suggests that the morphofunctional and physiological changes (or absence thereof) obtained in the experiment Intestinal tract organs under investigation in mice may also occur in humans under different environmental conditions [1-6].

\section{Experimental}

The experiment was carried out on white inbred mice of the $\mathrm{BALB} / \mathrm{c}$ line, the age of the animals 15 days. Throughout the experiment, mice were kept in standard vivar conditions, under natural light, without limiting access to food and water, in standard polycarbonate cages of five individuals each. Prior to the start of the study, all animals underwent a two-week adaptation period. For the experiment, the animals were randomized and divided into two groups of 15 individuals. Intact animals (group 1 - control) received standard granulated feed PC-120-1 (LLC "Laboratornas", Russia); animals of group 2 (experiment) were kept on the diet with the addition of

*Corresponding author; zyablova@mail.ru food additive. In an experiment to study the effect of an enriching additive on the clinical and biochemical status of laboratory animals, the body weight of mice in each group was determined daily, and the growth rate relative to the primary data was determined on the basis of the results obtained [7-10].

The blood taken from the tail vein before the beginning, on the 21 st day and after the end of the experiment was examined on a number of clinicalbiochemical indicators by sets of test-systems «Vital Development Corporation» (Russia, St. Petersburg).

The general protein was determined using the biurettic method according to the instructions for the diagnostic set [11-17]. In order to carry out quantitative analysis, finished reagents and test samples were applied according to the recommended scheme. The samples were mixed, incubated for 15 minutes at $18-25{ }^{\circ} \mathrm{C}$. Photometration was performed against the idle sample at a wavelength of $540 \mathrm{~nm}$.

Cholesterol level was controlled using enzymatic colorimetric method in accordance with instructions [1117]. Ready reagents and test samples were entered in accordance with the schematic in the instructions during the experiment. After mixing, the samples were incubated for 5 minutes at $18-25^{\circ} \mathrm{C}$. Photometration was performed against the idle sample at a wavelength of $500 \mathrm{~nm}$. Colour stability was maintained for at least 1 hour while being protected from direct sunlight.

The glucose content was determined using the glucose-oxidation method without deproteinization. In accordance with the instruction on the proposed scheme, quantitative analysis was performed [10-17]. The samples 
were mixed, incubated for 15 minutes at $18-25{ }^{\circ} \mathrm{C}$. Photometration was performed against the idle sample at a wavelength of $505 \mathrm{~nm}$. Colour stability was maintained for at least 1 hour while being protected from direct sunlight.

Low-density lipoproteins (LDL) were defined by an enzymatic colorimetric method with selective (no deposition) protection on the use of a diagnostic set [1117]. The test samples were incubated for 10 minutes at $18-25{ }^{\circ} \mathrm{C}$. Photometration was performed against the idle sample at a wavelength of $500 \mathrm{~nm}$. Next, the samples were centrifuged for 10 minutes at $4000 \mathrm{~g}$. Solutions for blank and calibration samples were not centrifuged. Transparent supernatant was used to determine the concentration of LDL. LDL was determined in all samples within an hour.

High density lipoproteins (HDL) were determined by an enzymatic method with immuno-inhibition without deposition in accordance with the procedure [11-17]. The samples were mixed and incubated for 5 minutes at 37 ${ }^{\circ} \mathrm{C}$, measuring adsorption A1, a sample of the idle sample. The samples were then re-mixed, incubated for 5 minutes at $37{ }^{\circ} \mathrm{C}$, adsorption $\mathrm{A} 2$ was measured. The difference of adsorptions $\Delta \mathrm{A}=\mathrm{A} 2-\mathrm{A} 1$ was then calculated. The LDP concentration in the sample (E) was then calculated with the formula:

$$
\mathrm{E}=\mathrm{E} \kappa \cdot \Delta \mathrm{Ao} / \Delta \mathrm{A} \kappa
$$

Where: $\mathrm{E} \kappa$ is calibrator concentration; $\Delta \mathrm{A} \kappa$ is adsorption difference of the calibrator; $\Delta \mathrm{Ao}$ is the difference in the adsorption of the experimental sample.

The phosphorus level was determined by UV method without deproteinization according to the instructions [11-17]. Experimental samples after mixing were incubated for 5 minutes at $18-25{ }^{\circ} \mathrm{C}$. Photometry was performed against an idle sample at a wavelength of 340 nm. The color stability was maintained for at least 24 hours while being protected from direct sunlight.

The calcium quantity was determined by the colorimetric method with Arsenazo III in accordance with the instructions for the application of the diagnostic set [11-17]. Complete reagents and test samples were added according to the recommended scheme. After mixing the samples were incubated for 5 minutes at 18$25{ }^{\circ} \mathrm{C}$. Photometration was performed against the idle sample at a wavelength of $340 \mathrm{~nm}$. Color stability was maintained for at least 1 hour while being protected from direct sunlight.

Alkaline phosphatase activity in serum and blood plasma was determined by an optimized kinetic method using the instruction [11-17]. A certain scheme was used for the application of finished reagents and test samples. This was followed by an incubation of experimental samples at a $37{ }^{\circ} \mathrm{C}$ water bath for 30 minutes. After the sample was cooled, appropriate reagents were introduced into the test tubes. The samples were then mixed and incubated for 5 minutes at $37{ }^{\circ} \mathrm{C}$. Photometry was performed against the idle sample at a wavelength of 405 $\mathrm{nm}$. The colour was stable for at least 8 hours in the absence of direct sunlight.

Statistical processing of the results of experimental studies was carried out using Statistic 6.0 software package for Windows using methods of descriptive statistics (mean error, standard deviation). The results are presented as $\mathrm{M} \pm \mathrm{SE}$. To determine the reliability of the obtained values, the Student T-test was used. The differences were considered significant when the value of the criterion is $\mathrm{p} \leqslant 0,05$ [18-19].

\section{Results and discussion}

The study of the influence of the food biopolymer system on the clinical and biochemical status of laboratory animals was carried out for 36 days. An in vivo experiment on white mice showed that the development of animals in the early stages took place without significant deviations in both groups. The safety of animals (experience and control) for the entire observation period was $100 \%$. This indicates the absence of toxic effects of feed on the organs and tissues of laboratory animals.

During the first 21 days of the experiment, the increase in body weight in groups 1 and 2 was $32.8 \%$. It was recorded that by day 36 the weight gain in animals of the experimental group was $17,6 \%$ higher than in animals of the intact group. This indicates a higher digestibility of feed containing an enriching additive compared to standard feed.

Clinical-biochemical indicators showed the normal flow of metabolic processes (Table 1).

Table 1. Results of research on exchange of macro and microelements of blood plasma clinical-biochemical status of laboratory animals in the invivo experiment

\begin{tabular}{|c|c|c|}
\hline \multirow{2}{*}{$\begin{array}{c}\text { Number of trial } \\
\text { days }\end{array}$} & \multicolumn{2}{|c|}{ Trial group } \\
\hline & 1 (Control) & 2 (Experiment) \\
\hline \multicolumn{3}{|c|}{ Glucose, $\mathrm{mmol} / \mathrm{l}$} \\
\hline 0 & \multicolumn{2}{|c|}{$4,5 \pm 0,21$} \\
\hline 21 & \multicolumn{2}{|c|}{$4,4 \pm 0,29$} \\
\hline 36 & $4,4 \pm 0,26$ & $4,5 \pm 0,82$ \\
\hline \multicolumn{3}{|c|}{ Total Protein, $\mathrm{g} / \mathrm{l}$} \\
\hline 0 & \multicolumn{2}{|c|}{$54,0 \pm 1,8$} \\
\hline 21 & \multicolumn{2}{|c|}{$55,0 \pm 2,0$} \\
\hline 36 & $54,0 \pm 1,3$ & $55,0 \pm 1,1$ \\
\hline \multicolumn{3}{|c|}{ Cholesterol level, $\mathrm{mmol} / 1$} \\
\hline 0 & \multicolumn{2}{|c|}{$2,2 \pm 0,10$} \\
\hline 21 & \multicolumn{2}{|c|}{$2,1 \pm 0,09$} \\
\hline 36 & $2,3 \pm 0,27$ & $2,2 \pm 0,28$ \\
\hline \multicolumn{3}{|c|}{ LDL, mmol/l: } \\
\hline 0 & \multirow{2}{*}{\multicolumn{2}{|c|}{$\begin{array}{l}0,44 \pm 0,05 \\
0,51 \pm 0,06\end{array}$}} \\
\hline 21 & & \\
\hline 36 & $2,46 \pm 0,12$ & $1,27 \pm 0,11$ \\
\hline \multicolumn{3}{|c|}{$\mathrm{HDL}, \mathrm{mmol} / \mathrm{l}$} \\
\hline 0 & \multicolumn{2}{|c|}{$0,71 \pm 0,14$} \\
\hline 21 & \multicolumn{2}{|c|}{$0,84 \pm 0,12$} \\
\hline 36 & $3,61 \pm 0,16$ & $2,96 \pm 0,15$ \\
\hline \multicolumn{3}{|c|}{ Phosphorus level, $\mathrm{mmol} / \mathrm{l}$} \\
\hline 0 & \multirow{2}{*}{\multicolumn{2}{|c|}{$\begin{array}{l}0,8 \pm 0,05 \\
0,7 \pm 0,04\end{array}$}} \\
\hline 21 & & \\
\hline 36 & $0,8 \pm 0,04$ & $0,9 \pm 0,04$ \\
\hline \multicolumn{3}{|c|}{ Calcium level, mmol/1 } \\
\hline 0 & \multicolumn{2}{|c|}{$1,9 \pm 0,13$} \\
\hline 21 & \multicolumn{2}{|c|}{$1,7 \pm 0,15$} \\
\hline 36 & $1,9 \pm 0,14$ & $2,1 \pm 0,16$ \\
\hline \multicolumn{3}{|c|}{ Alkaline Phosphatase level, $\mathrm{mcmol} / \mathrm{l}$} \\
\hline 0 & \multirow{2}{*}{\multicolumn{2}{|c|}{$\begin{array}{l}113,0 \pm 5,0 \\
116,0 \pm 7,0\end{array}$}} \\
\hline 21 & & \\
\hline 36 & $115,0 \pm 3,5$ & $115 \pm 3,5$ \\
\hline
\end{tabular}


The common protein of the experimental and control animal groups in all blood samples tested did not exceed the physiological standard and averaged 54-55 g/l for all groups. This indicates the normal flow of exchange processes in both pilot groups. Blood plasma cholesterol at the end of the experiment in the target animals was 4,5 $\%$ above the physiological norm, while the corresponding figure for group 2 was within the physiological norm. This shows that there is no negative effect of the feed with the bioactive additive on the lipid exchange of living organisms. The LDL level was also within the physiological norm (no more than $3 \mathrm{mmol} / \mathrm{L}$ ), and the HDL rate increased by an average of 4,6 times in all groups. This fact is not evidence of any negative processes, but primarily shows the presence of an active level of parietal digestion and lipid intake in the body [20-22].

The control of glucose content in both groups showed that its amount fluctuated slightly within the physiological norm. A slightly higher glucose content $(4,5 \pm 0,82 \mathrm{mmol} / \mathrm{L})$ was observed in blood plasma in experimental animal group 2, this is $2,3 \%$ higher than the blood glucose level of the animal control group at the end of the experiment. These data indicate a higher rate of assimilation of feed carbohydrates of groups of animals with enriched food systems [20-22].

The studies also monitored the exchange of some microelements. Results of experiments to exchange macro and microelements of plasma in mice in an in vivo experiment to study the effect of feedstuff enrichment on the clinical-biochemical status of animals in the experimental group showed that calcium and phosphorus were increased (by 12,5\% and 10,5\% respectively) compared to mice of the intact group (Table I). This confirms the higher availability and digestibility of microelements in feed containing a biopolymeric composition. Studies have found the same level of alkaline phosphatase against a background of stable microelements (calcium, phosphorus), indicating normal synthesis of collagen by osteoblasts under moderate activity of osteoclasts. This fact indicates the predominance of osteosynthesis in the bone tissue of animals that consumed food with bioadditive.

\section{Conclusion}

Studies conducted using bioassay in vivo techniques allow concluding that the developed food enrichment additive does not show any signs of toxicity and is biologically safe for the health of living organisms. It was found that a more significant increase in body weight of mice was recorded in the experimental group compared to the control group, which indicates a high digestibility of feed containing an enriching food additive. The dynamics of clinical and biochemical indicators shows not only the normal course of metabolic processes in laboratory animals, but also a higher level of carbohydrate absorption in the feed of the experimental group. In this group, calcium and phosphorus levels were higher than those of animals in the intact group, indicating the most frequent high availability and assimilation of micronutrients in the feed. The same level of alkaline phosphatase against the background of a stable content of trace elements confirms the normal synthesis of collagen by osteoblasts against the background of moderate activity of osteoclasts. This fact indicates the predominance of osteosynthesis processes in the bone tissue of experimental animals. The results obtained allow us to speak about the feasibility of introducing a developed biopolymer enriching additive into the composition of food products [23-27].

\section{References}

1. L.V. Antipova, N.S. Rodionov, and E.S. Popov, Journal of Higher Educational Institutions. Food Technology, 1, 8-11, (2018)

2. S.V. Belokurov, N.S. Rodionova, and E.V. BelokurovaJournal of physics, 1015, 32-107, (2018)

3. T.V. Alexeeva, Yu.O. Kaigina, and A.P. Fursova, Commodity expert of food products, 9, 69-77, (2019)

4. N.A. Zherebtsov, T.V. Zyablova, and T.N. Popova, Applied Biochemistry and Microbiology, 2, 145149, (2001)

5. D. Pass and G. Freeth, ANZCCART News, 4, 1-4, (1993)

6. M. Fitzgerald and J. Nolan P., J. Anat, 11, 67-71, (1971)

7. I.P. Zapadnuk, Laboratory animals. Breeding, maintenance, experimentation (Vysha shkola, Kiev, 1983)

8. E. Satinoff and I. Whishalow, The behavior of the laboratory rat. A handbook with tests (Oxford University Press, NY, 2005)

9. C. Gordon, Temperature Regulation in Laboratory Rodents (Cambridge University Press, N-Y, 1993)

10. V. Zutphen, V. Baumans, and A. Beynen, Principles of Laboratory Animal Science( Elsevier, Amsterdam, 2001)

11. Reference book. Biochemestry. Reagent kits for laboratory diagnostics (VostokMedBuisness, Khabarovsk, 2019)

12. M.A. Goryacheva and M.N. Makarova, International Veterinary Journal, 3, 155-159 (2016)

13. A. Babicky, Physi-ologia Bohemoslovaca, 35, 456463, (2006)

14. C. Argmann, M. Champy, and J. Auwerx, Curr. Protoc. Mol. Biol., 29, 27-73, (2007)

15. H. Donnelly, Laboratory Animals, 16, 167-171, (2002)

16. Y. Yorozu, M. Hirano, and K. Oka, Digests 9th Annual Conf. Magnetics Japan, 2, 740-741, (1987)

17. B. Klausen and H. Hougen, Laboratory Animals, 21, 324-347, (1987)

18. G.I. Nazarenko and A.A. Kishkun, Clinical evaluation of laboratory results. (Medecine, Moscow, 2000) 
19. E.Yu. Ludupova, N.V. Rinchinova, and R.V. Dugarova, Healthcare, 12, 80-88, (2015)

20. N.N. Karkishchenko and S.V. Grachev, Guidance on Laboratory and Alternative Models in Biomedical Technologies (Profile, Moscow, 2010)

21. Reference book. Physiological, biochemical and biometric standards of experimental animals ("LEMA" Publishing, Saint Petersburg, 2013)

22. Reference book. Farm animals. Physiological and biochemical parameters of the body (VNIIFBiP, Borovsk, 2002)

23. V.D. Bogdanov, O.V. Sazarova, and T.G. Saharova, Scientific papers of Dalrybvuz, 37, 93-98, (2016)

24. T.V. Alexeeva, A.A. Rodionov, and A.A. Vesnina "Economics. Innovations. Quality management, 3, 127-131, (2015)
25. L.Yu. Lazhentseva, University Journal. Food Technology, 1, 108-110, (2009)

26. E.V. Belokurova, N.S. Rodionova, and S.A. Solohin, Journal of Advanced research in technical science, 1, 2474-5901, (2016)

27. L.V. Golubeva, E.A. Pozhidaeva, and E.S. Popov, Indian Journal of scienct and technology, 42, 104224, (2016)

28. N.N. Alekhina, E.I. Ponomareva, and S.I. Lukina, Journal of engineering and applied sciences, 12, 2623-2627, (2016)

29. E.I. Ponomareva, V.I. Popov, and I.E. Esaulenko, Nutrition issues, 5, 75-81, (2017)

30. L.V. Golubeva, E.A. Pozhidaeva, and G.M. Smolsky, Food Industry, 1, 60-64, (2020) 